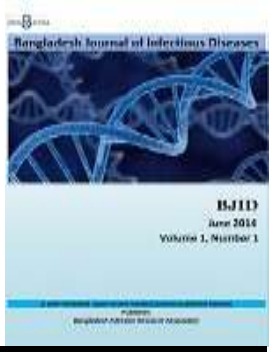

\title{
Melioidosis Mimicking Tuberculosis in an Endemic Zone: A Case Report
}

\author{
Prabhasish Adhikary ${ }^{1}$, Shahjada Selim ${ }^{2}$, Nazim Uddin ${ }^{3}$, Sajalendu Biswas ${ }^{4}$, Ariful Basher ${ }^{5}$, Hassan \\ Mahmoud $^{6}$, Md. Abdullah Yusuf ${ }^{7}$, Hafez Mohammad Nazmul Ahsan ${ }^{8}$, Syed Ghulam Mogni \\ Mowla $^{9}$, Md. Ridwanur Rahman ${ }^{10}$
}

${ }^{1}$ Registrar, Department of Medicine, Shaheed Suhrawardy Medical College Hospital, Dhaka, Bangladesh; ${ }^{2}$ Assistant Professor, Department of Endocrinology, Bangbandhu Sheikh Mujib Medical University, Dhaka. Bangladesh; ${ }^{3}$ Assistant Registrar, Department of Medicine, Shaheed Suhrawardy Medical College Hospital, Dhaka, Bangladesh; ${ }^{4}$ Medical Officer, Department of Medicine, Shaheed Suhrawardy Medical College \& Hospital, Dhaka. Bangladesh; ${ }^{5}$ Registrar, Department of Medicine, Mymensingh Medical College Hospital, Mymensingh, Bangladesh; ${ }^{6}$ Medical Officer, Department of Medicine, Shaheed Suhrawardy Medical College Hospital, Dhaka, Bangladesh; ${ }^{7}$ Assistant Professor, Department of Microbiology, National Institute of Neurosciences, Dhaka, Bangladesh; ${ }^{8}$ Assistant Professor, Department of Medicine, Shaheed Suhrawardy Medical College, Dhaka, Bangladesh; ${ }^{9}$ Assistant Professor, Department of Medicine, Shaheed Suhrawardy Medical College, Dhaka, Bangladesh; ${ }^{10}$ Professor \& Head, Department of Medicine, Shaheed Suhrawardy Medical College. Dhaka, Bangladesh

[Received: 1 December 2014; Accepted: 15 April 2015; Published: 1 June 2015]

\begin{abstract}
Melioidosis is not widely recognized in Bangladesh which is evident from the paucity of published reports on melioidosis from this region. Here, we summarize the clinical presentation, laboratory results, prevention and control policies and make important recommendations for patient management. A 35years-old diabetic male forest officer from Gazipur Sadar located north-west of Dhaka city got admitted to Shaheed Suhrawardy Medical College Hospital, Dhaka with history of recurrent fever for last 1 year. Initially the fever was high grade (upto $105^{\circ} \mathrm{F}$ ), intermittent nature and lasted for a few days to week. About five months back, the patient developed a parietal abscess over the left lumber region and was treated surgically accordingly. The patient then gradually recovered and remained afebrile for about one month. Fever again recurred, high grade, quotidian in nature and has been persistent for the last 3 months. The patient had also developed marked loss of appetite, altered bowel habit with occasional vomiting and lost about $15 \mathrm{~kg}$ of his body weight. The patient noticed profuse watery diarrhea for last 2 days and got himself admitted. On examination, the patient was found wasted, conscious but slow mentation, dehydrated and moderately anaemic. His pulse was $116 / \mathrm{min}$, blood pressure $80 / 60 \mathrm{~mm} \mathrm{Hg}$, respiration $24 / \mathrm{min}$ and oral temperature was $103^{\circ} \mathrm{F}$ but no palpable lymph nodes. The patient had moderate hepato-splenomegaly with left sided pleural effusion and bilateral depressed ankle jerks. Burkholderia pseudomallei was isolated and identified by blood and urine culture as well as with serological test. We should be more alert among the diabetic patients who are presented with fever with high ESR and neutrophilic leucocytosis, even if radiography or cytopathology is indicative of tuberculosis. [Bangladesh J Infect Dis 2015;2(1):23-26]
\end{abstract}

Key words: Melioidosis; tuberculosis; Burkholderia pseudomallei

How to cite this article: Adhikary P, Selim S, Uddin N, Biswas S, Basher A, Mahmoud H, Yusuf MA, Ahsan HMN, Mowla SGM, Rahman MR. Melioidosis Mimicking Tuberculosis in an Endemic Zone: A Case Report. Bangladesh J Infect Dis 2015;2(1):23-26

Correspondence: Dr. Ariful Basher, Registrar (Infectious \& Tropical Diseases), Mymensingh Medical College Hospital. Mymensingh, Bangladesh; Email: arifulbasher@yahoo.com

Competing interests: The authors declare that they have no competing interests.

Authors' contributions: RR designed the study with help of PA,SS, NU, SB and HM designed the protocol of investigation, set up the clinical evaluation, and conducted investigation on, respectively; AB,MAY, HMNA and SGMM were responsible for experimental design, isolation and data analysis. All other co-authors participated in collection and management of data. NU and PA wrote the manuscript. $\mathrm{AB}$ helped in manuscript revision. 


\section{Introduction}

Melioidosis is a clinical disease caused by the soil and water saprophytic bacteria named Burkholderia pseudomallei ${ }^{1}$. It is endemic in South-East Asia and Northern Australia ${ }^{2}$. It occurs in humans as well as in a wide variety of animals ${ }^{2}$. Infection occurs by inoculation through minute skin abrasions or by inhalation, especially in people who have direct contact with wet soil or surface water along with underlying predisposing factors such as diabetes mellitus, alcohol intake, chronic renal or lung disease or immunosuppression ${ }^{4}$.

Clinical presentation may be acute, subacute or chronic. Melioidosis can also present as a rapidly fatal septicemic illness. In chronic form, melioidosis can involve the lung, bones, joints, liver, spleen, lymph nodes, myocardium, brain and skin, thus simulating a variety of diseases, including cryptic tuberculosis ${ }^{5}$. It has been rightly referred to as the 'remarkable imitator' and 'the mimicker of maladies' by various authors ${ }^{6}$. Bangladesh is endemic for $\mathrm{TB}$ and patients are often presumptively treated for TB on the basis of clinical suspicion or radiological, haematological or cytopathological reports, even in the absence of a positive acid-fast bacilli (AFB) smear and/or culture $^{7-8}$. This present case was diagnosed as melioidosis at a tertiary care hospital in Dhaka city.

\section{Case Presentation}

A 35 years old diabetic male had been working in forest department presented with longstanding fever with variable duration that was as high as $105^{\circ} \mathrm{F}$ and intermittent in nature. About five month back he had a history of parietal abscess over the left loin that was treated by incision, drainage and oral antibiotics without any culture report. He recovered well and remained afebrile for about 1 month. Then fever again developed with high grade quotidian and has been persistent since then. He also noticed marked appetite lost, altered bowel habit with occasional vomiting and lost about $15 \mathrm{~kg}$ body weight in last 3 months. Due to illness, he shifted his job from the field to desk work and leave office for last 2 months. On query, he noticed chronic mild dry cough but denied any respiratory distress, chest pain, jaundice, urinary complaints or any close contact with TB patient. He has been diabetic for 8 years and reports good control with oral drugs. For the last 2 days prior to hospitalization he developed watery diarrhea, about 15 to 20 times per day without abdominal colic or visible blood. He became severely prostrated and got admitted for better management. There was no history of dental procedure, sore throat, gum bleeding or bone pain, pruritus or nodular swelling at any part of the body. He was treated with parenteral Ceftriaxone (1 gram) twice daily and oral Clindamycin $(300 \mathrm{mg})$ thrice daily for 14 days. He was also empirically treated with anti-malarial drugs (Tab. Quinine \& Tab. Coartem). He had been a forest officer working in the forests of Sylhet (6 years) and Khulna (1 year) before the last posting at Gazipur for last 2 years. On Examination, he was ill looking and apathetic. The patient was underweight (BMI 17.14), malnourished and moderately anaemic. Pulse (116 beats/ min) was regular but low volume; however, blood pressure $(80 / 60 \mathrm{~mm}$ of $\mathrm{Hg}$ ) was also low and temperature $\left(103^{0} \mathrm{~F}\right)$ was high. Liver was palpable which was firm, smooth nontender and was about 4 $\mathrm{cm}$ from right sub-costal margin with rounded inferior border. Spleen was also palpable, about 3 $\mathrm{cm}$ from left sub-costal margin along its long axis with rounded margin, firm consistency and nontender smooth surface. Bowel sound was increased with no hepatic bruit or splenic rub. Breath sound is diminished from left $6^{\text {th }}$ intercostals space with no added sound. Examination of other systems was revealed no abnormalities. Routine blood was done and found a high ESR (70 mm in $1^{\text {st }}$ hour); with a low haemoglobin (10.2 gm/dL), leukocytosis (TC $11,900 / \mathrm{cmm}$ ) and neutrophilia (Neutrophils $77.0 \%$ ). Peripheral blood film was shown polymorphonuclear (PMN) leukocytosis. Fasting blood sugar (14.5 mmol/L) was high. Liver function test revealed normal serum bilirubin $(0.4 \mathrm{mg} / \mathrm{dL})$, raised SGPT (61U/L) and SGOT (85U/L); however, serum total protein $(59 \mathrm{gm} / \mathrm{L})$ and serum albumin $(30 \mathrm{gm} / \mathrm{L})$ were normal. Serum electrolytes were found normal $(\mathrm{Na}+: 133 \mathrm{mmol} / \mathrm{L} ; \mathrm{K}+: 4.5 \mathrm{mmol} / \mathrm{L}$; $\left.\mathrm{Cl}^{-} 92 \mathrm{mmol} / \mathrm{L}\right)$. Kidney function test was also normal (blood urea $35 \mathrm{mg} / \mathrm{dL}$; serum creatinine $1.1 \mathrm{mg} / \mathrm{dL}$ ). Ultrasonography of whole abdomen showed abscess or haematoma at lumbar region with splenic space occupying lesions. Chest X-ray $\mathrm{P} / \mathrm{A}$ view showed consolidation with pleural effusion on left lung. PCR of blood showed MTBDNA-negative.

\section{Isolation \& Identification of Burkholderia} pseudomallei: Urine and blood specimens were collected for the isolation and identification of Burkholderia pseudomallei. Culture of urine was performed in the MacConkey's agar media after incubation for $24 \mathrm{~h}$ at $37^{\circ} \mathrm{C}$ and was produced pink color colony as because it is not lactose fermenter ${ }^{9}$. The blood was cultured on blood agar media with lytic centrifugation method which had produced tiny colony with crenated margin ${ }^{9}$. Gram staining was performed to see the morphology of the bacteria after taking from colony growth and had 
shown Gram negative rod with safety pin shaped bacteria $^{10}$. Colony in Nutrient agar media was colorless and no pyocyanin pigment had been produced $^{11}$. Oxidase tests were performed by spreading a linear smear from the single colony taken from MacConkey's agar media on filter paper impregnated with Oxidase reagent (Difco Laboratories, Michigan, USA) and a strong purple reaction was produced in the paper within 10 second $^{12}$. Pseudomonas aeruginosa was used as a positive control. Gentamicin susceptibilities were determined by demonstration of a zone of inhibition around a 10- $\mu$ g disk (Oxoid, Heidelberg, Australia) and were found resistant.[13] Further investigation was done to make it more confirmed like inoculation into SS agar media produced colony growth; incubation at $42^{0} \mathrm{C}$ was produced the colony growth ${ }^{13}$. An indirect ELISA for $\operatorname{IgM}$ antibody was done and had found a high titre in the blood which was 1:1600 dilution ${ }^{14}$. All agglutination reactions were conducted in a class II biological safety cabinet by gowned and gloved staff. Finally the patient was treated with parenteral Ceftazidime $2 \mathrm{gm}$ three times daily for 2 weeks followed by oral Co-trimoxazole $960 \mathrm{mg}$ two times daily and oral Doxycycline $100 \mathrm{mg}$ twice daily for 3 months. Other supportive treatment like subcutaneous Insulin was given as required. Patient was gradually improved and become completely cure in subsequent follow up.

\section{Discussion}

Chronic Melioidosis is one of the variant of it which often mimics tuberculosis ${ }^{2}$. Therefore, it is frequently treated with anti-tuberculosis drugs in an area where tuberculosis is endemic ${ }^{2}$. This patient was diabetic. It was reported that diabetes was the most common underlying risk factor ${ }^{1-4}$. In another study patient with diabetes mellitus has been reported to increase the relative risk of infection by up to 100 -folds in certain age groups in North-East Thailand $^{15}$. The estimated relative risk of melioidosis in diabetic patients was respectively 13.1 and 5.9 in Thailand and Australia ${ }^{3-4}$.

The patient was a forest officer working in the different districts of Bangladesh. Thus there is a direct contact with the wet soil and surface water where the etiologic agent of melioidosis is found. The relationship between contact with wet soil as well as the surface water and melioidosis has been well documented in different literatures ${ }^{1,3-4}$. Similarly rice farmers in Thailand is constituted majority of the melioidosis patients ${ }^{3-4}$. During investigation, chest $\mathrm{X}$-ray of the melioidosis patient showed consolidation with pleural effusion on left lung mimicking pulmonary tuberculosis ${ }^{16}$. Similarly pulmonary involvement is reported to be the most common form of melioidosis ${ }^{2}$. Acute pulmonary melioidosis is believed to occur following inhalation of the causative agent, especially during heavy monsoonal rain and winds. On the other hand the chronic form is usually occurred through percutaneous inoculation of Burkholderia pseudomallei in endemic areas ${ }^{3-4}$. Bangladesh is a tropical country having rain in 4 to 6 months leading to a suitable place for this bacterium to breed.

USG of whole abdomen showed abscess or haematoma at lumbar region with splenic space occupying lesions. In addition to that multiple foci of microabsess were detected in liver and also in both right and left kidney. Melioidotic liver abscess on CT or ultrasound characteristically shows the presence of small, multiple cavities, giving a 'Swisss cheese' or 'honeycomb' pattern, with involvement of other organs, commonly the spleen ${ }^{17}$. Similar to present study isolated splenic abscess has also been reported ${ }^{18}$. A high degree of suspicion is required and isolation in culture seems to be the only way of confirming the diagnosis of melioidosis. Timely and accurate identification of this organism in the laboratory is crucial. Conventionally, raised ESR indicates chronic clinical presentation, with sterile pus cultures with or without suggestive radiological findings, is believed to be of tubercular origin and is often treated like Tuberculosis. In a report Vidyalakshmi et $\mathrm{al}^{2}$ has mentioned that fever in a diabetic patient with neutrophilic leucocytosis and elevated ESR levels seem to be a strong indicator of melioidosis in this south-east Asian region.

Tests required for the isolation and identification of B. pseudomallei are relatively simple and do not involve the use of expensive media or equipment. Oxidase-positive, non-fermenting Gram-negative bacilli tend to be reported as Pseudomonas species, without further identification ${ }^{19}$. It has been speculate that the incidence of melioidosis is rising in Bangladesh; therefore, it becomes imperative to accurately speciate every Pseudomonas-like isolate, especially when isolated from blood, pus, tissue or exudates. At present, isolation in culture is the only definitive and specific diagnostic method available for melioidosis, which takes 3-4 days at the earliest ${ }^{2}$. However, to be more certain that the organism was indeed B. pseudomallei, we can do more specific test like API 20NE, agglutination with specific latex reagent, $16 \mathrm{~S}$ sequencing, specific 
PCR etc, that are not available in our setting. There is a definite need for the development of improved, rapid and reliable diagnostic tests to differentiate between melioidosis and tuberculosis.

This patient was treated with ceftriaxone $(1 \mathrm{gm})$ injection twice daily and clindamycin (300 $\mathrm{mg}$ ) thrice daily for 14 days without any clinical improvement. Similar to the present condition, it has been well documented that routine drugs used for treatment of Gram-negative sepsis are not useful for treatment of melioidosis, although the bacterium shows in vitro susceptibility to a large number of antimicrobials, including third generation cephalosporins $^{2}$. The recommended treatment is intravenous ceftazidime during the acute phase (2 weeks) followed by a combination of cotrimoxazole, chloramphenicol and doxycycline during the eradication phase like 3-6 months ${ }^{26}$. Other third generation cephalosporins, including cefotaxime and ceftriaxone, are not recommended despite their excellent in vitro susceptibility, as they have been associated with high mortality ${ }^{1,4}$.

\section{Conclusion}

Timely and accurate identification of this organism in the laboratory is crucial. This could avoid unnecessary treatment with toxic and expensive anti-tuberculosis drugs. Better awareness and a high index of suspicion among clinicians, microbiologists and public health personnel would go a long way in detecting larger number of cases, resulting in timely institution of appropriate treatment. The suspicion of $B$. pseudomallei should especially be raised when Pseudomonas-like isolates are resistant to aminoglycosides and/or colistin. Melioidosis disease is mimicking with suspected tuberculosis; it should be ruled out while instituting presumptive anti-tuberculosis treatment in areas where both diseases are prevalent like south East Asian region.

\section{References}

1. White NJ. Melioidosis. Lancet 2003; 361: 1715-1722

2. Vidyalakshmi K, Chakrapani M, Shrikala B, Damodar S, Lipika S, Vishal S. Tuberculosis mimicked by melioidosis. Interna J Tuberculosis Lung Dis 2008;12(10):1209-15
3. Currie BJ. Melioidosis: an important cause of pneumonia in residents of and travellers returned from endemic regions. Eur Respir J 2003; 22: 542-550

4. Cheng AC, Currie BJ. Melioidosis: epidemiology, pathophysiology and management. Clin Microbiol Rev 2005; 18: 383-416

5. John T J. Melioidosis, the mimicker of maladies. Indian J Med Res 2002; 119

6. Poe RH, Vassallo CL, Domm BM. Melioidosis: the remarkable imitator. Am Rev Respir Dis 1971;104:427-31

7. Lee $\mathrm{CH}$, Kim WJ, Yoo CG, et al. Response to empirical antituberculosis treatment in patients with sputum smearnegative presumptive pulmonary tuberculosis. Respiration 2005;72:369-74

8. Vanden-Brande P, Vernies T, Verwerft J, Van Bleyenber R, Vanhoenacker F, Demedts M. Impact of age and radiographic presentation on the presumptive diagnosis of pulmonary tuberculosis. Respir Med 2002; 96: 979-983

9. Wuthiekanun V, Dance D, Chaowagul W. et al. Blood culture techniques for the diagnosis of melioidosis. Europ J Clin Microbiol Infect Dis 1990;9:654-658

10. Goven JRW. Pseudomonas, Stenotrophomonas, Burkholderia. In: Mackie \& McCartney Practical Medical Microbiology; 14th Edition; Editors: Collee JG, Fraser AG, Marmion BP, Simmons A; Churchill Livingstone, Elsevier India Pvt. Ltd, 1999; pp.419

11. Dance DA, Wuthiekanun V, Naigowit P, White NJ. Identification of Pseudomonas pseudomallei in clinical practice: use of simple screening tests and API 20NE. J Clin Path 1989;42(6):645-48

12. Wuthiekanun V, Smith MD, Dance DAB, Walsh AL, Pitt TL, White NJ. Biochemical characteristics of clinical and environmental isolates of Burkholderia pseudomallei. J Med Microbiol 1996;45(6):408-12

13. Inglis TJJ, Merritt A, Chidlow G, Aravena-Roman M, Harnett G. Comparison of Diagnostic Laboratory Methods for Identification of Burkholderia pseudomallei. J Clin Microbiol 2005;43(5):2201-2206

14. Inglis TJJ, Rolim DB, Rodriguez JLN. Clinical Guideline for Diagnosis and Management of Melioidosis. Rev Inst Med trop S Paulo 2006;48(1):1-4

15. Suputtamongkol Y, Hall AJ, Dance DA, et al. The epidemiology of melioidosis in Ubon Ratchatani, northeast Thailand. Int J Epidemiol 1994;23:1082-90

16. Kibbler CC, Roberts CM, Ridgway GL, Spiro SG. Melioidosis in a patient from Bangladesh. Postgrad Med J 1991;67:764-66

17. Apisarnthanarak A, Apisarnthanarak P, Mundy LM. Computed tomography characteristics of Burkholderia pseudomallei liver abscess. Clin Infect Dis 2006;42:98993

18. Lin CY, Chen TC, Lu PL, Lin WR, Chen YH. Melioidosis presenting with isolated splenic abscesses: a case report. Kaohsiung J Med Sci 2007;23:417-421

19. Collee JG, Fraser AG, Marmion BP, Simmons A. (ed.). Mackie \& McCartney practical medical microbiology, 14th ed. Churchill Livingstone, New York, N.Y. 1996 Dance DAB. Melioidosis. Reviews Med Microbiol 1990; 1: $143-15$ 